\title{
An Error Estimate Uniform in Time for Spectral Galerkin Approximations for the Equations for the Motion of a Chemical Active Fluid
}

\author{
M.A. ROJAS-MEDAR and S.A LORCA
}

\begin{abstract}
We study error estimates and their convergence rates for a approximate solutions of spectral Galerkin type for the equations for the motion of a viscous chemical active fluid in a bounded domain. We find error estimates that are uniform in time and also optimal in the $L^{2}$-norm and $H^{1}$. norm. New estimates in the $H^{-1}$-norm are given.
\end{abstract}

\section{INTRODUCTION}

In this work we will study error estimates and their respective convergence rates for approximate solutions of spectral Galerkin type for the equations for the motion of a viscous chemical active fluid. These

1991 Mathematics Subject Classification: 65M60, 76D05, 65M15, 76R99

Servicio publicaciones Univ. Complutense. Madrid, 1995. 
equations are considered in a bounded domain $\Omega \subseteq \mathbb{R}^{3}$, with boundary $\Gamma$, in the time interval $[0,+\infty)$. To describe them, let $u(t, x) \in$ $\mathbb{R}^{3}, \tilde{\theta}(\mathrm{t}, \mathrm{x}) \in \mathbb{R}, \tilde{\psi}(\mathrm{t}, \mathrm{x}) \in \mathbb{R}$ and $p(t, x) \in \mathbb{R}$ denote respectively the unknown velocity, temperature, the concentration of material in the liquid, and the pressure at time $t \in[0, \infty)$, at point $x \in \Omega$. Then, the governing equations at level of Oberbeck-Boussinesq approximation are

$$
\left\{\begin{array}{l}
\frac{\partial u}{\partial t}+u . \nabla u-\Delta u+\nabla p=j+(\tilde{\theta}+\tilde{\psi}) g, \\
\frac{\partial \dot{\theta}}{\partial t}+(u . \nabla) \bar{\theta}-k_{\tilde{\theta}} \Delta \tilde{\theta}=f, \\
\frac{\partial \tilde{\psi}}{\partial t}+(u . \nabla) \tilde{\psi}-k_{\tilde{\psi}} \Delta \tilde{\psi}=h, \\
\operatorname{div} u=0
\end{array}\right.
$$

together with the following boundary and initial conditions

$$
\left\{\begin{array}{l}
u=0, \tilde{\theta}=\theta_{1}, \tilde{\psi}=\psi_{1} \quad \text { on }(0,+\infty) \times \Gamma, \\
u(0, x)=u_{0}(x), \tilde{\theta}(0, x)=\bar{\theta}_{0}(x), \tilde{\psi}(0, x)=\tilde{\psi}(x) \text { in } \Omega
\end{array}\right.
$$

Hеге, $j(t, x) \in \mathbb{R}^{3}, \mathrm{~g}(\mathrm{t}, \mathrm{x}) \in \mathbb{R}^{3}, \mathrm{f}(\mathrm{t}, \mathrm{x}), \mathrm{h}(\mathrm{t}, \mathrm{x}) \in \mathbb{R}$ are known external sources; $\nu>0$ is the viscosity of fluid, $k_{\bar{\theta}}$ and $k_{\bar{\psi}}$ are the termal and solute diffusity, respectively, $\theta_{1}$ and $\psi_{1}$ are known functions; $u_{0}, \tilde{\theta}_{0}$ and $\psi_{0}$ are given functions on the variable $x \in \Omega$.

The expressions $\Delta, \nabla$ and div denote the gradient, Laplacian and divergence operators, respectively (we also denote $\frac{\partial u}{\partial t}$ by $u_{t}$ or $\partial_{t} u$ ); the $i$ th component of $(u . \nabla) u$ is given by $[(u \cdot \nabla) u]_{i}=\sum_{j=1}^{3} u_{j} \frac{\partial}{\partial x_{j}} u_{i}$ and $(u \cdot \nabla) \phi=\sum_{j=1}^{3} u_{j} \frac{\partial \phi}{\partial x_{j}}$ for $\phi=\tilde{\theta}$ or $\tilde{\psi}$.

For the derivation and physical discussion of equation (1.1) see, for instance, Joseph [7].

We observe that this model of fluid includes as a particular case the classical Navier-Stokes, which has been much studied (see, for instance, the classical books by Ladyzhenkaya [9], Lions [10] and Temam [21] 
and the references there in). It also includes the classical Boussinesq's problem (or Bernard's problem), (in the case when chemical reactions are absent: $\bar{\psi} \equiv 0$ ) which has been investigated by several authors; see for instance Hishida [6], Korenev [8], Morimoto [12], Shinbrot and Kotorynski [20] and references therein.

Concerning system (1.1). Belov and Kapitanov [2] study the stability of the solutions of the system (1.1) with different boundary conditions. They used linearization and fixed point arguments.

More interessed in techniques directly related with numerical applications, Rojas-Medar and Lorca [15], [17] established the local and global existence of strong solutions of $(1.1),(1.2)$ by the spectral Galerkin methods (see Rojas-Medar and Lorca [15]; [17]; [18] and also the next section for the precise statements of the results). Here, the word spectral is used in the sense that the eigenfunctions of the associated Stokes and Laplacian operators are used as the approximation basis.

In this paper we are interessed in establishing error estimate, uniform in time and the convergence rates of these spectral approximations in several norms. But, before we describe our results, let us briefly comment related results.

In [13], Rautmann gave a systematic development of error estimates for the spectral Galerkin approximations to classical Navier-Stokes equations.

These error estimates are local in the sense that, they depend on functions that grow exponentially with time, and, as observed by Heywood [5], this is the best that can be expected without further assumptions on the stability of the solution being approximated. Working with the classical Navier-Stokes equations and assuming the uniform in time in the $L^{2}$-norm of the gradient of the velocity (which is usually obtained in global existence theorem) and the exponential stability in the $H^{1}$ norm of the solution (which is natural), in the same paper [5], Heywood was able to derive an optimal uniform in time error estimates for the velocity in the $H^{1}$-norm. Assuming exponential stability in the $L^{2}$-norm, Salvi in [19] proved an optimal uniform in time error estimate for the velocity in the $L^{2}$-norm also for the classical Navier-Stokes equations.

Also, without explicity assuming the $L^{2}$ (or $H^{1}$ ) - exponential stability (this being in general difficult to verify), Rojas-Medar and Boldrini 
[14] proved an uniform in time optimal error estimates for the spectral Galerkin approximations in the $H^{1}$ and $L^{2}$ norms, under requirement that the external force field has a mild form of decay (analogous requirements will be considered will be considered in this work).

Concerning system (1.1)-(1.2), in a previous work [16] the authors obtained a local in time optimal $L^{2}$ and $H^{1}$ error estimates.

In this work, we will generalized the results of [14] to system (1.1) - (1.2), and we obtain the optimal error estimates uniform in time.

The paper is organized as follows. In Section 2 we describe the approximations, we state certain known results that will be used later on in the paper, and we derive certain a priori estimates. In Section 3 we obtain our optimal $L^{2}$-estimate; in Section 4 we derive our, optimal $H^{1}$-estimate, and in Section 5 we present two new estimates in the $H^{-1}$. norm. We would like to say that this estimates are also true for the Navier-Stokes equations. Consequently, this estimates complements the results obtained earlier by Rautmann [13], Heywood [5], and RojasMedar and Boldrini [14].

We observe that, as it is usual, we will denote by $C$ a generic positive constant depending only on $\Omega$ and the data of the problem.

\section{PRELIMINARIES AND A PRIORI ESTIMATES}

We start by recalling certain definitions and facts that will be used in the rest of the paper.

In what follows we will assume $\Omega$ of class $C^{1,1}$. We will consider the usual Sobolev spaces

$$
W^{m, q}(D)=\left\{f \in L^{q}(D) ;\left\|\partial^{\alpha} f\right\|_{L^{q}(D)}<+\infty,(|\alpha| \leq m)\right\}
$$

$m=0,1,2, \ldots, 1 \leq q \leq \infty, D=\Omega$ or $(0, T) \times \Omega, 0<T \leq+\infty$, with the usual norm. When $q=2$, we denote $H^{m}(D)=W^{m, 2}(D)$ and $H_{0}^{m}(D)=$ closure of $C_{0}^{\infty}(\Omega)$ in $H^{m}(D)$. If $B$ is a Banach-space, we denote by $L^{q}(0, T, B)$ the Banach space of the $B$-valued functions defined in the interval $[0, T)$ that are $L^{q}$-integrable in the sense of Bochner. When $B=L^{p}(\Omega)$, we will denote $L^{q}\left((0, T) ; L^{p}(\Omega)\right)$ by $L^{q, p}((0, T) \times \Omega)$ for $1 \leq p, q \leq+\infty$. Also, we denote by $H^{-1}(\Omega)$ the topological dual of $H_{0}^{1}(\Omega)$. 
To easy the notation, in the rest of this paper the functions which are $\mathbb{R}$ or $\mathbb{R}^{3}$ valued will not be notationally distinguished; the distinction will be clear from the context.

Let $C_{o, \sigma}^{\infty}(\Omega)=\left\{v \in C_{0}^{\infty}(\Omega) ; \operatorname{div} v=0\right.$ in $\left.\Omega\right\} ; V=$ closure of $C_{0, \sigma}^{\infty}(\Omega)$ in $H_{0}^{1}(\Omega)$, and $H=$ closure of $C_{0, \sigma}^{\infty}(\Omega)$ in $L^{2}(\Omega), V^{*}=$ Topological dual of $V$.

Let $P$ be the orthogonal projection from $L^{2}(\Omega)$ onto $H$ obtained by the usual Helmholtz decomposition. Then the operator $A: H \rightarrow H$ given by $A=-P \Delta$ with domain $D(A)=H^{2}(\Omega) \cap V$ is called the Stokes operator. It is well known that $A$ is a positive definite self-adjoint operator and is characterized by the relation

$$
(A w, v)=(\nabla w, \nabla u) \text { for all } w \in D(A), v \in V
$$

From now on, we denote the inner product in $H$ (i.e., the $L^{2}$-inner product) by $($,$) . The general L^{p}$-norm will denoted by \|\|$_{L^{p}}$; to easy the notation, in the case $p=2$ we simply denote the $L^{2}$-norm by \|\| .

We observe that for the regularity properties of the Stokes operator, it is usually assumed that $\Omega$ is of class $C^{3}$; this being in order to use Cattabriga's results [3]. We use instead the stronger results of Amrouche and Girault [1] which implies, in particular, that when $A u \in\left(L^{2}(\Omega)\right)^{3}$ then $u \in H^{2}(\Omega)$ and $\|u\|_{H^{2}}$ and $\|A u\|$ are equivalent when $\Omega$ is of class $C^{1,1}$. This will be enough for all of the results in this paper.

The same remark is valid for the Laplacian operator $B=-\Delta$ : $L^{2}(\Omega) \rightarrow L^{2}(\Omega)$ with the Dirichlet boundary conditions with domain $D(B)=H_{0}^{1}(\Omega) \cap H^{2}(\Omega)$.

We shall denote by $w^{k}(x)$ and $\lambda_{k}$ the eigenfunctions and the eigenvalues of the Stokes operator. It is well known (see [4], [21]) that $w^{k}(x)$ are orthogonal in the inner product $(\cdot, \cdot),(\nabla u, \nabla v),(A u, A v)$ and complete in the spaces $H, V$ and $H^{2} \cap V$, respectively.

For each $k \in N$, we denote by $P_{k}$ the orthogonal projection from $L^{2}(\Omega)$ onto $V_{k}=\operatorname{span}\left[w^{1}, \ldots, w^{k}\right]$.

The following results can be found in Rautmann's paper [13]. 
Lemma 2.1. If $v \in V$, then holds

$$
\left\|v-P_{k} v\right\|^{2} \leq \frac{1}{\lambda_{k+1}}\|\nabla v\|^{2}
$$

Also, if $v \in V \cap H^{2}(\Omega)$, we have

$$
\begin{aligned}
& \left\|v-P_{k} v\right\|^{2} \leq \frac{1}{\lambda_{k+1}^{2}}\|A v\|^{2}, \\
& \left\|\nabla v-\nabla P_{k} v\right\|^{2} \leq \frac{1}{\lambda_{k+1}}\|A v\|^{2} .
\end{aligned}
$$

Further informations on the Stokes operator and spaces of divergence-free functions can be found in Constantin and Foias [4] and Temam [21].

Now, let us denote $B=-\Delta: L^{2}(\Omega) \rightarrow L^{2}(\Omega)$ with the Dirichlet boundary conditions with domain $D(B)=H_{0}^{1}(\Omega) \cap H^{2}(\Omega)$ and $\varphi^{k}(x), \gamma_{k}$ be the eigenfunctions and eigenvalues of $B$, respectively. As it is well known, all the above porperties have a corresponding one for $B$.

We will denote $R_{k}, k \in N$, the orthogonal projection of $L^{2}(\Omega)$ onto $H_{k}=\operatorname{span}\left[\varphi^{1}, \cdots, \varphi^{k}\right]$.

Before we give the definition of strong solution, we transform problem (1.1)-(1.2) into another problem with homogeneous boundary value. In order to do it, we consider extensions $\theta_{2}$ and $\psi_{2}$ of the functions $\theta_{1}$ and $\psi_{1}$, respectively, such that

$$
\begin{cases}\partial_{t} \theta_{2}-\Delta \theta_{2}=0 & ; \partial_{t} \psi_{2}-\Delta \psi_{2}=0 \text { in }(0, \infty) \times \Omega \\ \theta_{2}=\theta_{1} & ; \quad \psi_{2}=\psi_{1} \text { on }(0, \infty) \times \Gamma \\ \theta_{2}(0) \in H^{2}(\Omega) & ; \psi_{2}(0) \in H^{2}(\Omega)\end{cases}
$$

where $\theta_{2}(0)=\theta_{1}(0)$ on $\Gamma$ and $\psi_{2}(0)=\psi_{1}(0)$ on $\Gamma$. We known that problems (2.1) are uniquely solvable for suitable conditions for $\theta_{1}$ and 
$\psi_{1}$ (see Lions-Magenes [11] and references there in) with continuous dependence on the initial datas.

Now, we can transform the equations (1.1)-(1.2) by introducing the new variables $\theta=\tilde{\theta}-\theta_{2}$ and $\psi=\tilde{\psi}-\psi_{2}$, obtaining

$$
\begin{gathered}
\left\{\begin{array}{c}
\partial_{t} u+(u . \nabla) u-\Delta u+\nabla p=(\theta+\psi) g+g_{1} \\
\partial_{t} \theta+(u . \nabla) \theta-\Delta \theta=f-(u . \nabla) \theta_{2} \\
\partial_{t} \psi+(u . \nabla) \psi-\Delta \psi=h-(u . \nabla) \psi_{2} \\
\operatorname{div} u=0 \quad \text { in }(0, T) \times \Omega \\
u=0 ; \theta=0 ; \psi=0 \text { on }(0, T) \times \Gamma
\end{array}\right. \\
u(0)=u_{0} ; \theta(0)=\theta_{0} \equiv \tilde{\theta}_{0}-\theta_{2}(0) ; \psi(0)=\psi_{0} \equiv \tilde{\psi}_{0}-\psi_{2}(0)
\end{gathered}
$$

where $g_{1}=\left(\theta_{2}+\psi_{2}\right) g+j$. Here, without loosing generality for our purposes, we have put the viscosity and coefficients of diffusity to be one.

We observe that the problem (1.1)-(1.2) is equivalent to the problem (2.2)-(2.4); with this is mind, it is enough to study the problem (2.2)(2.4).

With the above notation, we write problem (2.2)-(2.4) as follows: find $(u, \theta, \psi) \in C\left([0, T] ; V \times\left(H_{0}^{1}(\Omega)^{2}\right) \cap L^{2}\left(0, T ; D(A) \times(D(B))^{2}\right)\right.$, $\left(\partial_{t} u, \partial_{t} \psi\right) \in L^{2}\left(0, T ; H \times\left(L^{2}(\Omega)\right)^{2}\right)(0<T \leq+\infty)$ such that

$$
\begin{aligned}
& u_{t}+P(u . \nabla u)+A u=P\left((\theta+\psi) g+g_{1}\right) \\
& \theta_{t}+u . \nabla \theta+B \theta=f-u . \nabla \theta_{2} \\
& \psi_{t}+u . \nabla \psi+B \psi=h-u . \nabla \psi_{2} \\
& u(0)=u_{0}, \theta(0)=\theta_{0}, \psi(0)=\psi_{0}
\end{aligned}
$$


which is equivalent to the weak form

$$
\begin{gathered}
\frac{d}{d t}(u, v)+(u . \nabla u, v)+(\nabla u, \nabla v)=\left((\theta+\psi) g+g_{1}, v\right), \forall v \in V \\
\frac{d}{d t}(\theta, \xi)+(u . \nabla \theta, \xi)+(\nabla \theta, \nabla \xi)=\left(f-u . \nabla \theta_{2}, \xi\right), \forall \xi \in H_{0}^{1} \\
\frac{d}{d t}(\psi, \phi)+(u . \nabla \psi, \phi)+(\nabla \psi, \nabla \phi)=\left(h-u . \nabla \psi_{2}\right), \forall \phi \in H_{0}^{1} \\
u(0)=u_{0}, \theta(0)=\theta_{0}, \psi(0)=\psi_{0} .
\end{gathered}
$$

The above functions $(u, \theta, \psi)$ are called strong solutions for the system $(2.2)-(2.4)$.

Concerning the existence of solutions for the above equations, one way to proceed is to use the Galerkin method. That is, we consider the Galerkin approximations: $u^{k}(x, t)=\sum_{i=1}^{k} a_{i k}(t) w^{i}(x), \theta^{k}(x, t)=$ $\sum_{i=1}^{k} b_{i k}(t) \varphi^{k}(x), \psi^{k}(x, t)=\sum_{i=1}^{k} c_{i k}(t) \varphi^{i}(x)$, satisfying the following equations

$$
\begin{gathered}
u_{t}^{k}+P_{k}\left(u^{k} \cdot \nabla u^{k}\right)+A u^{k}=P_{k}\left(\left(\theta^{k}+\psi^{k}\right) g+g_{1}\right), \\
\theta_{t}^{k}+R_{k}\left(u^{k} \cdot \nabla \theta^{k}\right)+B \theta^{k}=R_{k}\left(f-u^{k} \cdot \nabla \theta_{2}\right), \\
\psi_{t}^{k}+R_{k}\left(u^{k} \cdot \nabla \psi^{k}\right)+B \psi^{k}=R_{k}\left(h-u^{k} \cdot \nabla \psi_{2}\right), \\
u^{k}(0)=P_{k} u_{0}, \theta^{k}(0)=R_{k} \theta_{0}, \psi^{k}(0)=R_{k} \psi_{0},
\end{gathered}
$$

which is equivalent to the weak form

$$
\left(u_{t}^{k}, v\right)+\left(u^{k} \cdot \nabla u^{k}, v\right)+\left(\nabla u^{k}, \nabla v\right)=\left(\left(\theta^{k}+\psi^{k}\right) g+g_{1}, v\right), \quad \forall v \in V_{k}
$$




$$
\begin{gathered}
\left(\theta^{k}, \xi\right)+\left(u^{k} \cdot \nabla \theta^{k}, \xi\right)+\left(\nabla \theta^{k}, \nabla \xi\right)=\left(f-u^{k} \cdot \nabla \theta_{2}, \xi\right), \quad \forall \xi \in H_{k} \\
\left(\psi_{t}^{k}, \phi\right)+\left(u^{k} \cdot \nabla \psi^{k}, \phi\right)+\left(\nabla \psi^{k}, \nabla \phi\right)=\left(h-u^{k} \cdot \nabla \psi_{2}, \phi\right), \quad \forall \phi \in H_{k} \\
u^{k}(0)=P_{k} u_{0}, \quad \theta^{k}(0)=R_{k} \theta_{0}, \quad \psi^{k}(0)=R_{k} \psi_{0} .
\end{gathered}
$$

Then, it can be proved that $\left(u^{k}, \theta^{k}, \psi^{k}\right)$ converges is an appropriate sente to a solution $(u, \theta, \psi)$ of $(2.2)-(2.4)$ as $k \rightarrow \infty$. These conditions are given in the following Theorem, proved by Rojas-Medar and Lorca [17, Theorem 3.1 and Corollary 3.2] on the existence and uniqueness of global strong solutions for problem (2.2),(2.4).

Theorem 2.2. Let $u_{0} \in V, \theta_{0}, \psi_{0} \in H_{0}^{1}(\Omega), \theta_{2}, \psi_{2} \in L^{\infty}(0, \infty$; $\left.H^{1}(\Omega)\right) \cap L^{2}\left(0, T ; H^{1}(\Omega)\right), g \in L^{\infty, 3}((0, \infty) \times \Omega), j, f, h \in L^{\infty, 2}((0, \infty) \times$ $\Omega) \cap L^{2,2}((0, \infty) \times \Omega)$ If $\left\|u_{0}\right\|_{1},\left\|\theta_{0}\right\|_{1},\left\|\psi_{0}\right\|_{1},\left\|\theta_{2}\right\|_{L^{\infty}\left(0, \infty ; H^{2}(\Omega)\right)}$, $\left\|\psi_{2}\right\|_{L^{\infty}\left(0, \infty ; H^{2}(\Omega)\right)},\|f\|_{\left.L^{\infty}((0, \infty) \times \Omega)\right)},\|h\|_{L^{\infty, 2}((0, \infty) \times \Omega)}$, $\|j\|_{\left.L^{\infty, 2}(0, \infty) \times \Omega\right)}$ and $\|g\|_{L^{\infty, 3}((0, \infty) \times \Omega)}$ are sufficiently small, then the unique solution of (2.2)-(2.4) exists for all $t \geq 0$ and satisfies

$$
\begin{aligned}
& \sup _{t \geq 0}\{\|\nabla u(t)\|,\|\nabla \theta(t)\|,\|\nabla \psi(t)\|\}<+\infty \\
& \sup _{t \geq 0} \int_{0}^{t}\left(\|A u(s)\|^{2}+\|B \theta(s)\|^{2}+\|B \psi(s)\|^{2}\right) d s<+\infty \\
& \sup _{t \geq 0} \int_{0}^{t}\left(\left\|u_{t}(s)\right\|^{2}+\left\|\theta_{t}(s)\right\|^{2}+\left\|\psi_{t}(s)\right\|^{2}\right) d s<+\infty
\end{aligned}
$$

the same estimates hold the Galerkin approximations $u^{k}, \theta^{k}$ and $\psi^{k}$.

Corollary 2.3. Under the hypothesis of Theorem 2.2, there exists a positive constant $C$ such that for each $k \in \mathbb{N}$ and $t \geq 0$, we have 


$$
\begin{gathered}
\left\|u(t)-P_{k} u(t)\right\|^{2} \leq \frac{C}{\lambda_{k+1}}, \\
\int_{0}^{t}\left\|u(s)-P_{k} u(s)\right\|^{2} d s \leq \frac{C}{\lambda_{k+1}} .
\end{gathered}
$$

Proof. It immediately follows from Lemma 2.1, and the estimates of the Theorem 2.2 .

Remark. The above Corollary, also, is valid for $\theta$ and $\psi$, instead of $u$.

We will need, to obtain optimal rate of convergence, the following stronger Theorem, also proved in [17] (Theorem 3.3, p.13 and Corollary 3.4, p.16).

Theorem 2.4. The assumptions are those of Theorem 2.2 and we assume, moreover that $\partial_{t} \theta_{2}, \partial_{t} \psi_{2} \in L^{\infty, 2}((0, \infty) \times \Omega), \partial_{t} j, \partial_{t} f, \partial_{t} h \in$ $\left.L^{\infty, 2}((0, \infty) \times \Omega) \cap L^{2,2}((0, \infty) \times \Omega)\right) ; \partial_{t} g \in L^{\infty}((0, \infty) \times \Omega)$ and the initial data $u_{0} \in V \cap H^{2}(\Omega), \theta_{0}, \psi_{0} \in H_{0}^{1}(\Omega) \cap H^{2}(\Omega)$. Then, the solution obtained in Theorem 2.2 satisfies

$$
\begin{aligned}
& \sup _{t \geq 0}\left\{\left\|u_{t}(t)\right\|,\left\|\theta_{t}(t)\right\|,\left\|\psi_{t}(t)\right\|\right\}<+\infty ; \\
& \sup _{t \geq 0}\{\|A u(t)\|,\|B \theta(t)\|,\|B \psi(s)\|\}<+\infty ; \\
& \sup _{t \geq 0} \int_{0}^{t}\left(\left\|\nabla u_{t}(s)\right\|^{2}+\left\|\nabla \theta_{t}(s)\right\|^{2}+\left\|\nabla \psi_{t}(s)\right\|^{2}\right) d s<+\infty .
\end{aligned}
$$

The same estimates hold for the Galerkin approximations $u^{k}, \theta^{k}$ and $\psi^{k}$.

Corollary 2.5. Under the hypothesis of Theorem 2.4, there exists a positive constant $C$ such that for each $k \in \mathbb{N}$ and $t \geq 0$ we have 


$$
\begin{aligned}
& \left\|u(t)-P_{k} u(t)\right\|^{2} \leq \frac{C}{\lambda_{k+1}^{2}} \\
& \left\|\nabla u(t)-\nabla P_{k} u(t)\right\|^{2} \leq \frac{C}{\lambda_{k+1}} \\
& \int_{0}^{t}\left\|u(s)-P_{k} u(s)\right\|^{2} d s \leq \frac{C}{\lambda_{k+1}^{2}} \text { and } \\
& \int_{0}^{t}\left\|\nabla u(s)-\nabla P_{k} u(s)\right\|^{2} d s \leq \frac{C}{\lambda_{k+1}}
\end{aligned}
$$

Remark. Similar estimates are valid for $\theta$ and $\psi$.

\section{OPTIMAL $L^{2}$-ERROR ESTIMATES}

In this section an uniform in time optimal $L^{2}$-error estimates will be derived. The analysis will be done by using the following facts. Let $u=\sum_{i=1}^{\infty} A_{i}(t) w^{i}(x), \theta=\sum_{i=1}^{\infty} B_{i}(t) \varphi^{i}(x)$ and $\psi=\sum_{i=1}^{\infty} C_{i}(t) \varphi^{i}(x)$ the eigenfunctions expansion of $u, \theta$ and $\psi$, respectively.

Let $v^{k}=P_{k} u=\sum_{i=1}^{k} A_{i}(t) w^{i}(x), \rho^{k}=R_{k} \theta=\sum_{i=1}^{k} B_{i}(t) \varphi^{i}(x)$ and $\beta^{k}=R_{k} \psi=\sum_{i=1}^{k} C_{i}(t) \varphi^{i}(x)$ are the $k^{t h}$ partical sums of the series for $u, \theta$ and $\psi$, respectively, and let

$$
\begin{aligned}
& E^{k}=u^{k}-v^{k}, \quad \varepsilon^{k}=\theta-\rho^{k}, \quad \delta^{k}=\psi-\beta^{k} \\
& \eta^{k}=v^{k}-u^{k}, \quad r^{k}=\rho^{k}-\theta^{k}, \quad \alpha^{k}=\beta^{k}-\psi^{k}
\end{aligned}
$$

where $u^{k}, \theta^{k}$ and $\psi^{k}$ are the $k^{\text {th }}$ Galerkin approximations of $u, \theta$ and $\psi$, respectively. Then

$$
u-u^{k}=E^{k}+\eta^{k}, \quad \theta-\theta^{k}=\varepsilon^{k}+r^{k}, \quad \psi-\psi^{k}=\delta^{k}+\alpha^{k} .
$$


With these notations, we state.

Lemma 3.1. There exist a positive constant $C$, independent of $k \in \mathbb{N}$, such that

$$
\begin{aligned}
& \left\|\eta^{k}(t)\right\|^{2}+\left\|r^{k}(t)\right\|^{2}+\left\|\alpha^{k}(t)\right\|^{2}+\int_{0}^{t}\left(\left\|\nabla \eta^{k}(s)\right\|^{2}+\left\|\nabla r^{k}(s)\right\|^{2}+\right. \\
& \left.+\left\|\nabla \alpha^{k}(s)\right\|^{2}\right) d s \leq C\left(\frac{1}{\lambda_{k+1}}+\frac{1}{\gamma_{k+1}}\right) .
\end{aligned}
$$

Proof. We observe that $v^{k}, \rho^{k}$ and $\beta^{k}$ satisfies

$$
\begin{gathered}
v_{t}^{k}+A v^{k}+P_{k}(u . \nabla u)=P_{k}\left((\theta+\psi) g+g_{1}\right) \\
\rho_{t}^{k}+B \rho^{k}+R_{k}(u . \nabla \theta)=R_{k}\left(f-u . \nabla \theta_{2}\right) \\
\beta_{t}^{k}+B \beta^{k}+R_{k}(u . \nabla \psi)=R_{k}\left(h-u . \nabla \psi_{2}\right) \\
v^{k}(0)=P_{k} u_{0}, \quad \rho^{k}(0)=R_{k} \theta_{0}, \quad \beta^{k}(0)=R_{k} \psi_{0} .
\end{gathered}
$$

Substracting (2.5) from (3.1), (2.6) from (3.2) and (2.7) from (3.3), respectively, we obtain

$$
\begin{gathered}
\eta_{t}^{k}+A \eta^{k}=P_{k}\left(\varepsilon^{k}+r^{k}\right) g+P_{k}\left(\delta^{k}+\alpha^{k}\right) g \\
-P_{k}\left(E^{k} \cdot \nabla u^{k}\right)-P_{k}\left(\eta^{k} \cdot \nabla u^{k}\right) \\
-P_{k}\left(u \cdot \nabla E^{k}\right)-\left(P_{k}\left(u \nabla \eta^{k}\right),\right. \\
r_{t}^{k}+B r^{k}=-R_{k}\left(E^{k} \cdot \nabla \theta_{2}\right)-R_{k}\left(\eta^{k} \cdot \nabla \theta_{2}\right)-R_{k}\left(E^{k} \cdot \nabla \theta\right) \\
-R_{k}\left(\eta^{k} \cdot \nabla \theta\right)-R_{k}\left(u^{k} \cdot \nabla \varepsilon^{k}\right)-R_{k}\left(u^{k} \cdot \nabla r^{k}\right),
\end{gathered}
$$




$$
\begin{gathered}
\alpha_{t}^{k}+B \alpha^{k}=-R_{k}\left(E^{k} \cdot \nabla \psi_{2}\right)-R_{k}\left(\eta^{k} \cdot \nabla \psi_{2}\right)-R_{k}\left(E^{k} \cdot \nabla \psi\right) \\
-R_{k}\left(\eta^{k} \cdot \nabla \psi\right)-R_{k}\left(u^{k} \cdot \nabla \delta^{k}\right)-R_{k}\left(u^{k} \cdot \nabla \alpha^{k}\right), \\
\eta^{k}(0)=0, r^{k}(0)=0, \alpha^{k}(0)=0 .
\end{gathered}
$$

By taking the inner product in $\left(L^{2}(\Omega)\right)^{n}$ of identity (3.4) with $\eta^{k}$ and also the inner product in $L^{2}(\Omega)$ of (3.5), (3.6) with $r^{k}$ and $\alpha^{k}$, respectively, we get.

$$
\begin{aligned}
\frac{1}{2} \frac{d}{d t}\left\|\eta^{k}\right\|^{2}+\left.\left\|\nabla \eta^{k}\right\|\right|^{2} & =\left(\left(\varepsilon^{k}+r^{k}\right) g, \eta^{k}\right)+\left(\left(\delta^{k}+\alpha^{k}\right) g, \eta^{k}\right) \\
- & \left(E^{k} \cdot \nabla u^{k}, \eta^{k}\right)-\left(\eta^{k} \cdot \nabla u^{k}, \eta^{k}\right) \\
- & \left(u \cdot \nabla E^{k}, \eta^{k}\right)-\left(u \cdot \nabla \eta^{k}, \eta^{k}\right), \\
\frac{1}{2} \frac{d}{d t}\left\|r^{k}\right\|^{2}+\left\|\nabla r^{k}\right\|^{2} & =-\left(E^{k} \cdot \nabla \theta_{2}, r^{k}\right)-\left(\eta^{k} \cdot \nabla \theta_{2}, r^{k}\right) \\
& -\left(E^{k} \cdot \nabla \theta, r^{k}\right)-\left(\eta^{k} \cdot \nabla \theta, r^{k}\right) \\
& -\left(u^{k} \cdot \nabla \varepsilon^{k}, r^{k}\right)-\left(u^{k} \cdot \nabla r^{k}, r^{k}\right) \\
\frac{1}{2} \frac{d}{d t}\left\|\alpha^{k}\right\|^{2}+\left\|\nabla \alpha^{k}\right\|^{2} & =-\left(E^{k} \cdot \nabla \psi_{2}, \alpha^{k}\right)-\left(\eta^{k} \cdot \nabla \psi_{2}, \alpha^{k}\right) \\
& -\left(E^{k} \cdot \nabla \psi, \alpha^{k}\right)-\left(\eta^{k} \cdot \nabla \psi, \alpha^{k}\right) \\
& -\left(u^{k} \cdot \nabla \delta^{k}, \alpha^{k}\right)-\left(u^{k} \cdot \nabla \alpha^{k}, \alpha^{k}\right) .
\end{aligned}
$$

We observe that

$$
\left(u . \nabla \eta^{k}, \eta^{k}\right)=\left(u^{k} \cdot \nabla r^{k}, r^{k}\right)=\left(u^{k} \cdot \nabla \alpha^{k}, \alpha^{k}\right)=0
$$


Also, by the Hölder and Young's inequalities we have

$$
\begin{aligned}
& \left|\left(\left(\varepsilon^{k}+r^{k}\right) g, \eta^{k}\right)\right| \leq C\left(\left\|r^{k}\right\|^{2}+\left.\left\|\varepsilon^{k}\right\|\right|^{2}\right)\|g\|_{L^{3}}^{2}+\frac{1}{10}\left\|\nabla \eta^{k}\right\|^{2} \\
& \left|\left(\left(\delta^{k}+\alpha^{k}\right) g, \eta^{k}\right)\right| \leq C\left(\left\|\delta^{k}\right\|^{2}+\left\|\alpha^{k}\right\|^{2}\right)\|g\|_{L^{3}}^{2}+\frac{1}{10}\left\|\nabla \eta^{k}\right\|^{2} .
\end{aligned}
$$

The Hölder's and Young's inequalities together with the Sobolev embedding $H^{1} \hookrightarrow L^{6}, H^{1} \hookrightarrow L^{3}$ imply

$$
\begin{aligned}
& \left|\left(E^{k} \cdot \nabla u^{k}, \eta^{k}\right)\right| \leq C\left\|A u^{k}\right\|^{2}\left\|E^{k}||^{2}+\frac{1}{10}\right\| \nabla \eta^{k} \|^{2} \\
& \left|\left(\eta^{k} \cdot \nabla u^{k}, \eta^{k}\right)\right| \leq C\left\|A u^{k}\right\|^{2}\left\|\eta^{k}\right\|^{2}+\frac{1}{10}\left\|\nabla \eta^{k}\right\|^{2} \\
& \left|\left(u . \nabla E^{k}, \eta^{k}\right)\right|=\left|\left(u . \nabla \eta^{k}, E^{k}\right)\right| \leq C\|A u\|^{2}\left\|E^{k}\right\|^{2}+\frac{1}{10}\left\|\nabla \eta^{k}\right\|^{2} .
\end{aligned}
$$

Analogously, we can prove that

$$
\begin{aligned}
& \left|\left(E^{k} . \nabla \theta_{2}, r^{k}\right)\right| \leq C\left\|E^{k}\right\|^{2}\left\|\nabla \theta_{2}\right\|_{L^{3}}^{2}+\frac{1}{10}\left\|\nabla r^{k}\right\|^{2}, \\
& \left|\left(\eta^{k} . \nabla \theta_{2}, r^{k}\right)\right| \leq C\left\|\eta^{k}\right\|^{2}\left\|\nabla \theta_{2}\right\|_{L^{3}}^{2}+\frac{1}{10}\left\|\nabla r^{k}\right\|^{2}, \\
& \left|\left(E^{k} \cdot \nabla \theta, r^{k}\right)\right| \leq C\left\|E^{k}\right\|^{2}\|B \theta\|^{2}+\frac{1}{10}\left\|\nabla r^{k}\right\|^{2}, \\
& \left|\left(\eta^{k} \cdot \nabla \theta, r^{k}\right)\right| \leq C\left\|\eta^{k}\right\|^{2}\|B \theta\|^{2}+\frac{1}{10}\left\|\nabla r^{k}\right\|^{2},
\end{aligned}
$$


$\left|\left(u^{k} . \nabla \varepsilon^{k}, r^{k}\right)\right|=\left|\left(u^{k}, \nabla r^{k}, \varepsilon^{k}\right)\right| \leq C\left\|\varepsilon^{k}\right\|^{2}\left\|A u^{k}\right\|^{2}+\frac{1}{10}\left\|\nabla r^{k}\right\|^{2}$.

Analogous estimates are satisfied by $\alpha^{k}$.

Bearing in mind the above estimates and the differential equalities (3.7)-(3.9), we obtain the integral inequality

$$
\begin{aligned}
& \left\|\eta^{k}(t)\right\|^{2}+\left\|\sigma^{k}(t)\right\|^{2}+\left\|\alpha^{k}(t)\right\|^{2}+\int_{0}^{t}\left(\left\|\nabla \eta^{k}(s)\right\|^{2}+\left\|\nabla r^{k}(s)\right\|^{2}+\right. \\
& \left.\left\|\nabla \alpha^{k}(s)\right\|^{2}\right) d s \leq \int_{0}^{t} \chi(s)\left(\left\|\eta^{k}(s)\right\|^{2}+\left\|r^{k}(s)\right\|^{2}+\left\|\alpha^{k}(s)\right\|^{2}\right) d s+ \\
& \int_{0}^{t} \chi(s)\left(\left\|E^{k}(s)\right\|^{2}+\left\|\varepsilon^{k}(s)\right\|^{2}+\left\|\delta^{k}(s)\right\|^{2}\right) d s,
\end{aligned}
$$

where $\chi(s)=c\left(\|g(s)\|_{L^{3}}^{2}+\left\|\nabla \theta_{2}(s)\right\|_{L^{3}}^{2}+\left\|\nabla \psi_{2}(s)\right\|_{L^{3}}^{2}+\|A u(s)\|^{2}+\right.$ $\left.\|B \theta(s)\|^{2}+\|B \psi(s)\|^{2}+\left\|A u^{k}(s)\right\|^{2}\right)$.

Now, by applying Gronwall's inequality, we obtain

$$
\begin{aligned}
& \left\|\eta^{k}(t)\right\|^{2}+\left\|r^{k}(t)\right\|^{2}+\left\|\alpha^{k}(t)\right\|^{2}+\int_{0}^{t}\left(\left\|\nabla \eta^{k}(s)\right\|^{2}+\left\|\nabla r^{k}(s)\right\|^{2}+\right. \\
& \left.\left\|\nabla \alpha^{k}(s)\right\|^{2}\right) d s \leq \int_{0}^{t} \chi(s)\left(\left\|E^{k}(s)\right\|^{2}+\left\|\varepsilon^{k}(s)\right\|^{2}+\right. \\
& +\left\|\delta^{k}(s)\right\| d s \exp \int_{0}^{t} \chi(s) d s .
\end{aligned}
$$


By using the estimates given in the Lemma 2.1, we have

$$
\begin{aligned}
& \int_{0}^{t} \chi(s)\left(\left\|E^{k}(s)\right\|^{2}+\left\|\varepsilon^{k}(s)\right\|+\left\|\delta^{k}(s)\right\|^{2}\right) d s \exp \int_{0}^{t} \chi(s) d s \\
\leq & \left(\frac{1}{\lambda_{k+1}}+\frac{2}{\gamma_{k+1}}\right) \int_{0}^{t} \chi(s) d s \exp \int_{0}^{t} \chi(s) d s .
\end{aligned}
$$

Now, by the estimates given in the Theorem 2.2, we have

$$
\int_{0}^{t} \chi(s) d s \leq C, \quad \text { for all } t \geq 0
$$

This, together with (3.10) and (3.11), implies the result.

Now, we are ready to prove the following

Theorem 3.2. Suppose the assumptions of the Theorem 2.2 hold. Then, the approximations $u^{k}, \theta^{k}$ and $\psi^{k}$ satisfy

$\left\|u(t)-u^{k}(t)\right\|^{2}+\left\|\theta(t)-\theta^{k}(t)\right\|^{2}+\left\|\psi(t)-\psi^{k}(t)\right\|^{2} \leq C\left(\frac{1}{\lambda_{k+1}}+\frac{1}{\gamma_{k+1}}\right)$ for all $t \geq 0$.

Proof. We have

$$
\begin{aligned}
\left\|u(t)-u^{k}(t)\right\|^{2} & \leq\left\|u(t)-v^{k}(t)\right\|^{2}+\left\|v^{k}(t)-u^{k}(t)\right\|^{2} \\
& \leq\left\|E^{k}(t)\right\|^{2}+\left\|\eta^{k}(t)\right\|^{2} \\
& \leq C\left(\frac{1}{\lambda_{k+1}}+\frac{1}{\gamma_{k+1}}\right)
\end{aligned}
$$

thanks to the Lemma 3.1 and Corollary 2.3 Similar results can be proved for $\theta^{k}$ and $\psi^{k}$. 
By using the Theorem 2.4 and Corollary 2.5, we prove the following stronger result

Theorem 3.3. Suppose the assumption of the Theorem 2.4 hold. Then, the approximations $u^{k}, \theta^{k}$ and $\psi^{k}$, satisfies

$$
\begin{aligned}
& \left\|u(t)-u^{k}(t)\right\|^{2}+\left\|\theta(t)-\theta^{k}(t)\right\|^{2}+\left\|\psi(t)-\psi^{k}(t)\right\|^{2} \\
& +\int_{0}^{t}\left(\left\|\nabla u(s)-\nabla u^{k}(s)\right\|^{2}+\left\|\nabla \theta(s)-\nabla \theta^{k}(s)\right\|^{2}+\right. \\
& \left.\quad+\left\|\nabla \psi(s)-\nabla \psi^{k}(s)\right\|^{2}\right) d s \leq C\left(\frac{1}{\lambda_{k+1}^{2}}+\frac{1}{\gamma_{k+1}^{2}}\right)
\end{aligned}
$$

for all $t \geq 0$.

Analogously as in the proof of the Theorem 3.2 , and with the same notation, we only have to proof the following.

Lemma 3.4. There exist a positive constante $C$, such that

$$
\begin{aligned}
& \left\|\eta^{k}(t)\right\|^{2}+\left\|r^{k}(t)\right\|^{2}+\left\|\alpha^{k}(t)\right\|^{2}+\int_{0}^{t}\left(\left\|\nabla \eta^{k}(s)\right\|^{2}+\left\|\nabla r^{k}(s)\right\|^{2}+\right. \\
& \left.+\left\|\nabla \alpha^{k}(s)\right\|^{2}\right) d s \leq C\left(\frac{1}{\lambda_{k+1}^{2}}+\frac{1}{\gamma_{k+1}^{2}}\right)
\end{aligned}
$$

for all $t \geq 0$.

Proof. By using the Lemma 3.1, and the estimates given is the Theorem 2.2 and the inequality (3.11), we have the required result.

\section{OPTIMAL $H^{1}$-ERROR ESTIMATES UNIFORM IN TIME}

In this section an uniform in time optimal $H^{1}$ error estimate will be derived. 
Theorem 4.1. Under the hypothesis of the Theorem 2.4 , we have that there exists a constante $C>0$

$$
\begin{aligned}
& \left\|\nabla u(t)-\nabla u^{k}(t)\right\|^{2}+\left\|\nabla \theta(t)-\nabla \theta^{k}(t)\right\|^{2}+\left\|\nabla \psi(t)-\nabla \psi^{k}(t)\right\|^{2} \\
& +\int_{0}^{t}\left(\left\|u_{t}(s)-u_{t}^{k}(s)\right\|^{2}+\left\|\theta_{t}(s)-\theta_{t}^{k}(s)\right\|^{2}+\left\|\psi_{t}(s)-\psi_{t}^{k}(s)\right\|^{2}\right) d s \\
& \quad \leq C\left(\frac{1}{\lambda_{k+1}}+\frac{1}{\gamma_{k+1}}\right)
\end{aligned}
$$

for all $t \geq 0$.

Analogously as in the section 3 , and with the same notation, we only have to prove the following

Lemma 4.2.

$$
\begin{aligned}
& \left\|\nabla \eta^{k}(t)\right\|^{2}+\left\|\nabla r^{k}(t)\right\|^{2}+\left\|\nabla \alpha^{k}(t)\right\|^{2}+\int_{0}^{t}\left(\left\|\eta_{t}^{k}(s)\right\|^{2}+\left\|r_{t}^{k}(s)\right\|^{2}+\right. \\
& +\left\|\alpha_{t}^{k}(s)\right\|^{2} d s \leq C\left(\frac{1}{\lambda_{k+1}}+\frac{1}{\gamma_{k+1}}\right)
\end{aligned}
$$

for all $t \geq 0$.

Proof. By taking the inner product in $\left(L^{2}(\Omega)\right)^{3}$ of identity (3.4) with $\eta_{t}^{k}$ and the inner product in $L^{2}(\Omega)$ of (3.5), (3.6) with $r_{t}^{k}$ and $\alpha_{t}^{k}$, repectively, we obtain

$$
\begin{aligned}
\frac{1}{2} \frac{d}{d t}\left\|\nabla \eta^{k}\right\|^{2}+\left\|\eta_{t}^{k}\right\|^{2} & =\left(\left(\varepsilon^{k}+r^{k}\right) g, \eta_{t}^{k}\right)+\left(\left(\delta^{k}+\alpha^{k}\right) g, \eta_{t}^{k}\right) \\
& -\left(E^{k} \cdot \nabla u^{k}, \eta_{t}^{k}\right)-\left(\eta^{k} \cdot \nabla u^{k}, \eta_{t}^{k}\right) \\
& -\left(u . \nabla E^{k}, \eta_{t}^{k}\right)-\left(u . \nabla \eta^{k}, \eta_{t}^{k}\right)
\end{aligned}
$$




$$
\begin{aligned}
\frac{1}{2} \frac{d}{d t}\left\|\nabla r^{k}\right\|^{2}+\left\|r_{t}^{k}\right\|^{2} & =-\left(E^{k} \cdot \nabla \theta_{2}, r_{t}^{k}\right)-\left(\eta^{k} \cdot \nabla \theta_{2}, r_{t}^{k}\right) \\
& -\left(E^{k} \cdot \nabla \theta, r_{t}^{k}\right)-\left(\eta^{k} \cdot \nabla \theta, r_{t}^{k}\right) \\
& -\left(u^{k} \cdot \nabla \varepsilon^{k}, r_{t}^{k}\right)-\left(u^{k} \cdot \nabla r^{k}, r_{t}^{k}\right), \\
\frac{1}{2} \frac{d}{d t}\left\|\nabla \alpha^{k}\right\|^{2}+\left\|\alpha_{t}^{k}\right\|^{2} & =-\left(E^{k} \cdot \nabla \psi_{2}, \alpha_{t}^{k}\right)-\left(\eta^{k} \cdot \nabla \psi_{2}, \alpha_{t}^{k}\right) \\
& -\left(E^{k} \cdot \nabla \psi, \alpha_{t}^{k}\right)-\left(\eta^{k} \cdot \nabla \psi, \eta_{t}^{k}\right) \\
& -\left(u^{k} \cdot \nabla \delta^{k}, \alpha_{t}^{k}\right)-\left(u^{k}, \nabla \alpha^{k}, \alpha_{t}^{k}\right) .
\end{aligned}
$$

We estimate the right-hand side of (4.1), (4.2) and (4.3) of the following manner. From the Hölder and Young inequalities, and by using the Sobolev embedding $H^{1} \hookrightarrow L^{6}, H^{2} \hookrightarrow L^{\infty}$, we have

$$
\begin{aligned}
& \left.\mid\left(\varepsilon^{k}+r^{k}\right) g, \eta_{t}^{k}\right) \mid \leq C\left(\left\|\nabla \varepsilon^{k}\right\|^{2}+\left\|\nabla r^{k}\right\|^{2}\right)\|g\|_{L^{3}}^{2}+\frac{1}{12}\left\|\eta_{t}^{k}\right\|^{2}, \\
& \left.\mid\left(\delta^{k}+\alpha^{k}\right) g, \eta_{t}^{k}\right) \mid \leq C\left(\left\|\nabla \delta^{k}\right\|^{2}+\left\|\nabla \alpha^{k}\right\|^{2}\right)\|g\|_{L^{3}}^{2}+\frac{1}{12}\left\|\eta_{t}^{k}\right\|^{2}, \\
& \left|\left(E^{k} \cdot \nabla u^{k}, \eta_{t}^{k}\right)\right| \leq C\left\|\nabla E^{k}\right\|^{2}\left\|A u^{k}\right\|^{2}+\frac{1}{12}\left\|\eta_{t}^{k}\right\|^{2}, \\
& \left|\left(\eta^{k} \cdot \nabla u^{k}, \eta_{t}^{k}\right)\right| \leq C\left\|\nabla \eta^{k}\right\|^{2}\left\|A u^{k}\right\|^{2}+\frac{1}{12}\left\|\eta_{t}^{k}\right\|^{2},
\end{aligned}
$$




$$
\begin{aligned}
& \left|\left(u . \nabla E^{k}, \eta_{t}^{k}\right)\right| \leq C\|A u\|^{2}\left\|\nabla E^{k}\right\|^{2}+\frac{1}{12}\left\|\eta_{t}^{k}\right\|^{2}, \\
& \left|\left(u . \nabla \eta^{k}, \eta_{t}^{k}\right)\right| \leq C\left\|\nabla \eta^{k}\right\|^{2}\|A u\|^{2}+\frac{1}{12}\left\|\eta_{t}^{k}\right\|^{2}, \\
& \left|\left(E^{k} . \nabla \theta_{2}, r_{t}^{k}\right)\right| \leq C\left\|\nabla E^{k}\right\|^{2}\left\|\nabla \theta_{2}\right\|_{L^{3}}^{2}+\frac{1}{12}\left\|r_{t}^{k}\right\|^{2}, \\
& \left|\left(\eta^{k} . \nabla \theta_{2}, r_{t}^{k}\right)\right| \leq C\left\|\nabla \eta^{k}\right\|^{2}\left\|\nabla \theta_{2}\right\|_{L^{3}}^{2}+\frac{1}{12}\left\|r_{t}^{k}\right\|^{2}, \\
& \left|\left(E^{k} . \nabla \theta, r_{t}^{k}\right)\right| \leq C\left\|\nabla E^{k}\right\|^{2}\|B \theta\|^{2}+\frac{1}{12}\left\|r_{t}^{k}\right\|^{2}, \\
& \left|\left(\eta^{k} \cdot \nabla \theta, r_{t}^{k}\right)\right| \leq C\left\|\nabla \eta^{k}\right\|^{2}\|B \theta\|^{2}+\frac{1}{12}\left\|r_{t}^{k}\right\|^{2}, \\
& \left|\left(u^{k} . \nabla \varepsilon^{k}, r_{t}^{k}\right)\right| \leq C\left\|A u^{k}\right\|^{2}\left\|\nabla \varepsilon^{k}\right\|^{2}+\frac{1}{12}\left\|r_{t}^{k}\right\|^{2}, \\
& \left|\left(u^{k} . \nabla r^{k}, r_{t}^{k}\right)\right| \leq C\left\|A u^{k}\right\|^{2}\left\|\nabla r^{k}\right\|^{2}+\frac{1}{12}\left\|r_{t}^{k}\right\|^{2} .
\end{aligned}
$$

The estimates for the terms that involve $\alpha^{k}$ are quite similar.

The above estimates, together with (4.1)-(4.3), imply

$$
\begin{aligned}
& \frac{d}{d t}\left(\left\|\nabla \eta^{k}\right\|^{2}+\left.\left\|\nabla r^{k}\right\|\right|^{2}+\left\|\nabla \alpha^{k}\right\|^{2}\right)+\left\|\eta_{t}^{k}\right\|^{2}+\left\|r_{t}^{k}\right\|^{2}+\left\|\alpha_{t}^{k}\right\|^{2} \\
& \leq \chi(t)\left(\left\|\nabla \eta^{k}\right\|^{2}+\left\|\nabla r^{k}\right\|^{2}+\left\|\nabla \alpha^{k}\right\|^{2}\right)+ \\
& +\chi(t)\left(\left\|\nabla E^{k}\right\|^{2}+\left\|\nabla \varepsilon^{k}\right\|^{2}+\left\|\nabla \delta^{k}\right\|^{2}\right)
\end{aligned}
$$

where $\chi(t)=C\left(\|g(t)\|_{L^{3}}^{2}+\left\|\nabla \theta_{2}\right\|_{L^{3}}^{2}+\left\|\nabla \psi_{2}(t)\right\|_{L^{3}}^{2}+\|A u(t)\|^{2}\right.$ 
$\left.+\|B \theta(t)\|^{2}+\|B \psi(t)\|^{2}+\left\|A u^{k}(t)\right\|^{2}\right)$.

Now, by the estimates given in the Theorem 2.4 we get

$$
\int_{0}^{t} \chi(s) d s \leq C, \quad \forall t \geq 0
$$

and consequently, by using the Gronwall's Lemma,

$$
\begin{aligned}
& \left\|\nabla \eta^{k}(t)\right\|^{2}+\left\|\nabla r^{k}(t)\right\|^{2}+\left\|\nabla \alpha^{k}(t)\right\|^{2} \\
& +\int_{0}^{t}\left(\left\|\eta_{t}^{k}(s)\right\|^{2}+\left\|r_{t}^{k}(s)\right\|^{2}+\left\|\alpha_{t}^{k}(s)\right\|^{2}\right) d s \\
& \leq \int_{0}^{t} \chi(s)\left(\left\|\nabla E^{k}(s)\right\|^{2}+\left\|\nabla \varepsilon^{k}(s)\right\|^{2}+\left\|\nabla \delta^{k}(s)\right\|^{2}\right) d s \exp C \\
& \leq \tilde{C}\left(\frac{1}{\lambda_{k+1}}+\frac{1}{\gamma_{k+1}}\right)
\end{aligned}
$$

thanks to the estimates given in the Lemma 2.1. This completes the proof.

Also, we can easily obtain

Proposition 4.3. Under the hypothesis of the Theorem 2.4, we have that there exists a positive constant $C$, such that

$$
\begin{aligned}
& \int_{0}^{t}\left(\left\|A u(s)-A u^{k}(s)\right\|^{2}+\left\|B \theta(s)-B \theta^{k}(s)\right\|^{2}\right. \\
& \left.+\left\|B \psi(s)-B \psi^{k}(s)\right\|^{2}\right) d s \leq C\left(\frac{1}{\lambda_{k+1}}+\frac{1}{\gamma_{k+1}}\right) .
\end{aligned}
$$

for all $t \geq 0$. 


\section{FURTHER HIGHER ORDER ESTIMATES}

In this section an uniform in time optimal $V^{*}$ error estimate (resp. $H^{-1}$ ) will be derived for $u_{t}$ (resp. $\theta_{t}$ and $\psi_{t}$ ) and $A u$ (resp. $B \theta$ and $B \psi)$.

Theorem 5.1. Under the hypothesis of the Theorem 2.4, we have that there exists a positive constant $C$, such that

$$
\begin{aligned}
& \left\|u_{t}(t)-u_{t}^{k}(t)\right\|_{V^{*}}^{2} \leq C\left(\frac{1}{\lambda_{k+1}}+\frac{1}{\gamma_{k+1}}\right) \\
& \left\|\theta_{t}(t)-\theta_{t}^{k}(t)\right\|_{H^{-1}}^{2} \leq C\left(\frac{1}{\lambda_{k+1}}+\frac{1}{\gamma_{k+1}}\right) \\
& \left\|\psi_{t}(t)-\psi_{t}^{k}(t)\right\|_{H^{-1}}^{2} \leq C\left(\frac{1}{\lambda_{k+1}}+\frac{1}{\gamma_{k+1}}\right)
\end{aligned}
$$

for all $t \geq 0$.

Proof. We observe that $u-u^{k}$ satisfies

$$
\begin{aligned}
u_{t}-u_{t}^{k}+A\left(u-u^{k}\right) & =P_{k}\left(\theta-\theta^{k}\right) g+P_{k}\left(\psi-\psi^{k}\right) g \\
& +\left(P-P_{k}\right)(\theta+\psi) g+\left(P-P_{k}\right) g_{1} \\
& \equiv F^{k}
\end{aligned}
$$

Consequently,

$$
\begin{aligned}
\left\|u_{t}-u_{t}^{k}\right\|_{V^{*}} & =\sup _{\|v\|_{v} \leq 1}\left|\left(F^{k}, v\right)+\left(A\left(u^{k}-u\right), v\right)\right| \\
& \leq \sup _{\|v\|_{v \leq 1}}\left|\left(F^{k}, v\right)\right|+\sup _{\|v\|_{v} \leq 1}\left|\left(\nabla\left(u-u^{\dot{k}}\right), \nabla v\right)\right| .
\end{aligned}
$$


On the other hand, we have

$$
\begin{aligned}
\left|\left(\nabla\left(u-u^{k}\right), \nabla v\right)\right| & \leq\left\|\nabla\left(u-u^{k}\right)\right\|\|\nabla v\| \\
& \leq C\left(\frac{1}{\lambda_{k+1}}+\frac{1}{\gamma_{k+1}}\right)^{1 / 2}\|v\|_{V},
\end{aligned}
$$

thanks to the Theorem 4.1.

Also,

$$
\begin{aligned}
\left|\left(F^{k}, v\right)\right| & \leq\left|\left(\left(\theta-\theta^{k}\right) g, P_{k} v\right)\right|+\left|\left(\left(\psi-\psi^{k}\right) g, P_{k} v\right)\right| \\
& +\left|\left((\theta+\psi) g,\left(P-P_{k}\right) v\right)\right|+\left|\left(g_{1},\left(P-P_{k}\right) v\right)\right|
\end{aligned}
$$

Since $v \in V$, Lemma 2.1 and Theorem 2.4, imply

$$
\begin{aligned}
\mid\left((\theta+\psi) g,\left(P-P_{k}\right) v \mid\right. & \leq\|\varphi+\psi\|_{L^{\boldsymbol{\varepsilon}}}\|g\|_{L^{3}} \frac{\|\nabla v\|}{\lambda_{k+1}^{1 / 2}} \\
& \leq C(\|\nabla \theta\|+\|\nabla \psi\|)\|g\|_{L^{3}} \frac{\|\nabla v\|}{\lambda_{k+1}^{1 / 2}} \\
& \leq \frac{C\|v\|_{v}}{\lambda_{k+1}^{1 / 2}}
\end{aligned}
$$

Analogously,

$$
\mid\left(g_{1},\left(P-P_{k}\right) v \mid \leq \frac{C}{\lambda_{k+1}^{1 / 2}}\|v\|_{V}\right.
$$


The other terms in the inequality (5.7) are estimated as follows

$$
\begin{aligned}
\left|\left(\left(\theta-\theta^{k}\right) g, P_{k} v\right)\right| & \leq\left\|\theta-\theta^{k}\right\| L_{L^{6}}\|g\|_{L^{3}}\left\|P_{k} v\right\| \\
& \leq C\left\|\nabla \theta-\nabla \theta^{k}\right\|\|g\|_{L^{3}}\|v\| \\
& \leq C\left(\frac{1}{\lambda_{k+1}}+\frac{1}{\gamma_{k+1}}\right)^{1 / 2}\|v\|_{V}
\end{aligned}
$$

where we have use the estimates given in the Theorem 4.1 , and the Sobolev embedding $H^{1} \hookrightarrow L^{6}$ and $V \hookrightarrow L^{2}$, and $\left\|P_{k}\right\|=1$.

Similarly, we obtain

$$
\left|\left(\left(\psi-\psi^{k}\right) g, P_{k} v\right)\right| \leq C\left(\frac{1}{\lambda_{k+1}}+\frac{1}{\gamma_{k+1}}\right)^{1 / 2}\|v\|_{V}
$$

The above inequalities together with (5.6) imply (5.1).

Now, we prove (5.2); The equalities for $\theta$ and $\theta^{k}$ imply

$$
\begin{aligned}
\theta_{t}-\theta_{t}^{k}+B\left(\theta-\theta^{k}\right) & =\left(I-R_{k}\right)\left(f-u \cdot \nabla \theta_{2}\right) \\
& +R_{k}\left(u-u^{k}\right) \cdot \nabla \theta^{2} \\
& \equiv G^{k}
\end{aligned}
$$

Consequently,

$$
\begin{aligned}
& \left\|\theta_{t}-\theta_{t}^{k}\right\|_{H^{-1}}=\sup _{\|\varphi\|_{H_{0}^{1}} \leq 1}\left|\left(G^{k}, \varphi\right)+\left(B\left(\theta-\theta^{k}\right), \varphi\right)\right| \\
& \leq \sup _{\|\varphi\|_{H_{0}^{1}} \leq 1}\left|\left(G^{k}, \varphi\right)\right|+\sup _{\|\varphi\|_{H_{0}^{1}} \leq 1}\left|\left(\nabla\left(\theta-\theta^{k}\right), \nabla \varphi\right)\right| .
\end{aligned}
$$


On the other hand, we have

$$
\begin{aligned}
\left|\left(\nabla\left(\theta^{k}-\theta\right), \nabla \varphi\right)\right| & \leq\left\|\nabla\left(\theta^{k}-\theta\right)\right\|\|\nabla \varphi\| \\
& \leq C\left(\frac{1}{\lambda_{k+1}}+\frac{1}{\gamma_{k+1}}\right)^{1 / 2}\|\varphi\|_{H_{0}^{1}}
\end{aligned}
$$

by Theorem 4.1. Also,

$$
\begin{aligned}
& \left|\left(R_{k}\left(u-u^{k}\right) \cdot \nabla \theta_{2}, \varphi\right)\right| \leq\left|\left(\left(u-u^{k}\right) \cdot \nabla \theta_{2}, R_{k} \varphi\right)\right| \\
& \leq\left.\left\|u-u^{k}\right\|\right|_{L^{4}}|| \nabla \theta_{2}\left\|_{L^{4}}\right\| R_{k} \varphi \| \\
& \leq C\left\|\nabla u-\nabla u^{k}\right\|\left\|B \theta_{2}\right\|\|\varphi\| \\
& \leq C\left(\underline{\frac{1}{\lambda_{k+1}}}+\frac{1}{\gamma_{k+1}}\right)^{1 / 2}\|\varphi\|_{H_{0}^{1}} .
\end{aligned}
$$

The other term in (5.8) is estimated as follows

$$
\begin{aligned}
\left|\left(\left(I-R_{k}\right)\left(f-u . \nabla \theta_{2}\right), \varphi\right)\right| & =\left|\left(f-u . \nabla \theta_{2},\left(I-R_{k}\right) \varphi\right)\right| \\
& \leq\left\|f-u . \nabla \theta_{2}\right\| \frac{\|\nabla \varphi\|}{\gamma_{k+1}^{1 / 2}} \\
& \leq \frac{C}{\gamma_{k+1}^{1 / 2}}\left(\|f\|+\|A u\|\left\|\nabla \theta_{2}\right\|\right)\|\varphi\|_{H_{0}^{1}}
\end{aligned}
$$


where we have used the estimates given in theorem 2.4 and Lemma 2.1. This and inequalities (5.10)-(5.11) imply

$$
\left\|G^{k}\right\|_{H^{-1}} \leq C\left(\frac{1}{\lambda_{k+1}}+\frac{1}{\gamma_{k+1}}\right)^{1 / 2}\|\varphi\|_{H_{\circ}^{1}}
$$

This estimate, together with (5.9), implies (5.2).

Finally, (5.3) is proved exactly as the above estimate.

Also, we have

Theorem 5.2. Under the hypothesis of the Theorem 2.4, we have that there exists a positive constant $C$, such that

$$
\begin{aligned}
& \left\|A u(t)-A u^{k}(t)\right\|_{V^{*}}^{2} \leq C\left(\frac{1}{\lambda_{k+1}}+\frac{1}{\gamma_{k+1}}\right) \\
& \left\|B \theta(t)-B \theta^{k}(t)\right\|_{H^{-1}}^{2} \leq C\left(\frac{1}{\lambda_{k+1}}+\frac{1}{\gamma_{k+1}}\right) \\
& \left\|B \psi(t)-B \psi^{k}(t)\right\|_{H^{-1}}^{2} \leq C\left(\frac{1}{\lambda_{k+1}}+\frac{1}{\gamma_{k+1}}\right)
\end{aligned}
$$

for all $t \geq 0$. proved.

Proof. We will prove (5.12). The other estimates are analogously Inequality (5.4) implies

$$
\left\|A\left(u-u^{k}\right)\right\|_{V^{*}} \leq\left\|u_{t}-u_{t}^{k}\right\|_{V^{*}}+\left\|F^{k}\right\|_{V^{*}} .
$$

By using the above theorem, we have the desired result.

\section{References}

[1] Amrouche, C. and Girault, V., On the existence and regularity of the solution of Stokes problem in arbitrary dimension, Proc. Japan Acad., 67, Ser. A (1991), 171-175. 
[2] Belov, V. Ya and Kapitonov, B.V., A certain hydrodinamic model of chemically active fluid, Sibirskii Matematicheskii Zhurnal, 24 (1983), 3-13.

[3] Cattabriga, L., Su un problema al contorno relativo al sistema di equazioni di Stokes, Rend. Mat. Univ. Padova 31 (1961), 308-340.

[4] Constantin, P. and Foias C., Navier-Stokes Equation, Chicago Lect. in Math., The University of Chicago Press, Chicago and London, 1989.

[5] Heywood, J.G., An error estimate uniform in time for spectral Galerkin approximations of the Navier-Stokes problem, Pac. J. Math. 96 (1982), 333-345.

[6] Hishida, T., Existence and regularizing properties of solutions for the nonstationary convection problems, Funcl. Ekav., 34 (1991), 449-474.

[7] Joseph, D.D., Stability of Fluid Motion, Springer-Verlag, Berlin, 1976.

[8] Korenev, N.K., On some problems of convection in a viscous incompressible fluid, Vestrik Liningrad Univ. Math., 4 (1977), 125-137.

[9] Ladyzhenskaya, O.A., The Mathematical Theory of Viscous Incompressible Flow, Gordon and Breach, Second Revised Edition, New York, 1969.

[10] Lions, J.L., Quelques Méthodes de Résolution des Problèmes Nonlinéaires, Dunod, Paris, 1969.

[11] Lions, J.L. and Magenes, Problemes aux limites Non Homogeneous et Applications, Vol II, Dunod, Paris, 1968.

[12] Morimoto, H., Non-stationary Boussinesq equations, J. Fac. Sci. Univ. Tokyo, Sect 1A, Math. 39 (1992), 61-75.

[13] Rautmann, R., On the convergence rate of Nonstationary NavierStokes Approximations, Proc. IUTAM Symp. 1979, Approx. Methods for Navier-Stokes Problem, R. Rautmann (Ed.), Springer-Verlag, Lect. Notes in Math., 771 (1980), 235-248.

[14] Rojas-Medar, M.A. and Boldrini, J.L., Spectral Galerkin approximations for the Navier-Stokes equations uniform in time error estimates, to appear in Rev. Mat. Apl. Vol 14, No2. 
[15] Rojas-Medar, M.A. and Lorca, S.A., The equations of viscous incompressible chemical active fluid: on the existence and regularity, Conferencia in the IV Simposio Chileno de Matemática, Santiago, Univ. de Santiago, Chile, 1993.

[16] Rojas-Medar, M.A. and Lorca, S.A., On the convergence rate of spectral aproximations for the equations for chemical active fluid, Relatório de Pesquisa, 23 (1993), IMECC-UNICAMP, Brazil; Submettid.

[17] Rojas-Medar, M.A., and Lorca, S.A., Global solution to the equations for the motion of a chemical active fluid, conference in the III Workshop in partial differential equations and applications, Rio de Janeiro, IMPA, Brazil, 1993.

[18] Rojas-Medar, M.A. and Lorca, S., In preparation.

[19] Salvi, R., Error estimates for the spectral Galerkin approximations of the solutions of Navier-Stokes type equation, Glasgow Math. J., 31 (1987), 199-211.

[20] Shinbrot, M. and Kotorynski, W.P., The initial value problem for a viscous heat conducting fluid, J. Math. Anal. Appl., 45 (1974), 1-22.

[21] Temam, R., Navier-Stokes Equations, Theory and Numerical Analysis, North-Holland, Second edition, Amsterdam, 1979.

Brazil. 